University of Wollongong

Research Online

Faculty of Engineering - Papers (Archive)

Faculty of Engineering and Information

Sciences

2003

\title{
On the limiting mechanism of irradiation enhancement of I/sub c/
}

A. Gandini

University of Houston, USA

R. Weinstein

University of Houston, USA

D. Parks

University of Houston, USA

R. Sawh

University of Houston, USA

S. X. Dou

University of Wollongong, shi@uow.edu.au

Follow this and additional works at: https://ro.uow.edu.au/engpapers

Part of the Engineering Commons

https://ro.uow.edu.au/engpapers/54

\section{Recommended Citation}

Gandini, A.; Weinstein, R.; Parks, D.; Sawh, R.; and Dou, S. X.: On the limiting mechanism of irradiation enhancement of I/sub c/ 2003.

https://ro.uow.edu.au/engpapers/54

Research Online is the open access institutional repository for the University of Wollongong. For further information contact the UOW Library: research-pubs@uow.edu.au 


\title{
On the Limiting Mechanism of Irradiation Enhancement of $I_{c}$
}

\author{
Alberto Gandini, Roy Weinstein, Drew Parks, Ravi P. Sawh, and Shi Xue Dou
}

\begin{abstract}
Irradiation may significantly increase $I_{c}$ in HTS. A systematic pattern occurs: $R=I_{c}$ (after irr.) $/ I_{c}$ (before irr.) increases at low defect density, $d$. It reaches a peak, and then it falls below 1 at high $d$.

The pinning center mechanism, which causes $R$ to increase, has been extensively studied. The falloff in $R$ has not. It has been considered a secondary effect.

Here, we will show that the fall-off plays an important role in determining the maximum $I_{c}$ enhancement achievable.

A phenomenological model to describe the $R$-vs.- $d$ curve, over the entire $d$ range, is proposed. The idea is that $R$ is the product of two competing effects. (i) Irradiation damage acts as pinning centers, hence increases critical current density, $J_{c}$. (ii) Damage reduces the flow-area. Hence, it decreases the net critical current.

Data on U/n processed Bi-2223 tapes are fitted to this model. The fitting indicates: (1) the reduction of the flow-area accounts for the majority of the $R$ falloff; and (2) It is sufficient to describe $J_{c}$ enhancement as linear with $d$, and it depending on field and temperature only through the ratio $b=B / B_{\text {irr }}$, where $B_{\text {irr }}$ is the irreversible field before irradiation.
\end{abstract}

Index Terms-Critical current density, high temperature superconductors, pinning centers, radiation effects.

\section{INTRODUCTION}

$\mathbf{O}$ VER the past decade, the effects of radiation on HTS have been extensively studied. Two main lines of research can be identified.

On one hand, there are studies [1]-[5] on the morphology of the defects, the behavior of the HTS critical temperature, $T_{c}$, and the HTS normal state resistivity, and their dependence upon irradiation dose and energy. These studies show that amorphous regions are randomly positioned within the HTS, and that radiation-induced defects decrease $T_{c}$.

On the other hand, interest is focused on interaction between vortices and radiation-damage, and the enhancement of $J_{c}$ by the irradiation-induced pinning centers [6]-[8].

These studies show that a systematic pattern occurs: irradiation increases $I_{c}$ at low radiation-induced defect density, $d$. Then, $I_{c}$ enhancement saturates, it reaches a peak, and then it falls-off below the pre-irradiation values at high $d$.

Manuscript received August 5, 2002. This work was support in part by the U.S. Army Research Office, the Welch Foundation, the State of Texas via the Texas Center for Superconductivity and Advance Material (TCSAM), and the Australian Research Council.

A. Gandini, R. Weinstein, D. Parks, and R. P. Sawh are with the Department of Physics and Texas Center for Superconductivity and Advance Material (TCSAM), University of Houston, Houston, TX 77204-5005 USA (e-mail: Agandini@UH.EDU).

S. X. Dou is with the Institute of Superconducting and Electronic Materials, University of Wollongong, NSW, Australia.

Digital Object Identifier 10.1109/TASC.2003.812058
The $I_{c}$ fall-off is usually attributed mostly to the reduction of $T_{c}$, and the fall-off at high $d$ has been considered as an inevitable secondary effect. For this reason, most of the studies have been carried at low radiation fluence, below the peak of $I_{c}$ attainable.

Recently, we began to address this matter [9], and realized that to further improve $I_{c}$ a deeper understanding of the mechanisms that limit $I_{c}$ enhancement, causing $R$ to fall-off, is of fundamental importance. In particular, it would be valuable to be able to quantify this effect.

In this paper, we begin to address the following questions. What causes $I_{c}$ to decrease at high fluence? Can the decrease be quantify and directly related to the type and energy of the radiation? Is there a way that $I_{c}$ may be increased above the today's limit [10]?

Section II describes the experimental set-up of an experiment on $\mathrm{Ag} / \mathrm{Bi}-2223$ tapes. In Section III, the $I_{c}$ enhancement in $\mathrm{U} / \mathrm{n}$ $\mathrm{Ag} / \mathrm{Bi}-2223$ tapes processed with fission-ions (U/n process) is presented. These data serve as a starting point of a phenomenological model proposed in Section IV. This model is fitted to the data; the results are discussed in Section V. Conclusions are drown in Section VI.

\section{EXPERIMENTAL SET-UP}

We applied the U/n process [11] to $\mathrm{Ag} / \mathrm{Bi}-2223$ tape [12]. In the $\mathrm{U} / \mathrm{n}$ process, uranium $\left({ }^{235} \mathrm{U}\right)$ is added to the HTS precursor powder. After processing by PIT process [13], samples are irradiated with thermal neutrons. Through thermal neutron irradiation some of the ${ }^{235} \mathrm{U}$ atoms fission [14]. Fission products create short quasicolumnar defects, circa $2.7 \mu \mathrm{m}$ long and $3.8 \mathrm{~nm}$ in diameter [9], [11] (3.8 $\mathrm{nm}$ is the diameter of the amorphous area, the net damaged area is about twice the amorphous one). The quasicolumnar defects act as pinning centers and improve $I_{c}$ [9], [11], [12], [15].

In this experiment, uranium was added in several concentrations: $0.15 \%, 0.4 \%, 0.6 \%$, and $1.0 \%$ by wt. of $\mathrm{UO}_{4}$. The thermal neutron fluences used ranged over 2 orders of magnitude, from $3.12 \times 10^{14}$ to $3.62 \times 10^{16} \mathrm{n} / \mathrm{cm}^{2}$. The density of fission fragments density, $d\left(\mathrm{~cm}^{-3}\right)$, is given by:

$$
d=\frac{2 \sigma \rho N_{a} x F_{n}}{A},
$$

where $F_{n}$ is the thermal neutron fluence, $x$ is the percent weight of ${ }^{235} \mathrm{U}, \sigma$ is the ${ }^{235} \mathrm{U}$ fission cross section, $\rho \sim 6 \mathrm{~g} / \mathrm{cm}^{3}$ is the Bi-2223 density, $N_{a}$ is the Avogadro's number, and $A$ is the ${ }^{235} \mathrm{U}$ atomic mass. From the above equation the fission fragment density is found to range between: $\sim 5 \times 10^{12}$ to $\sim 6 \times 10^{15} \mathrm{~cm}^{-3}$.

The critical current, $I_{c}$, was measured by means of the fourpoint technique. Each sample was measured, before and after irradiation, at temperatures between $65 \mathrm{~K}$ and $77 \mathrm{~K}$, and up to $5 \mathrm{~T}$ 


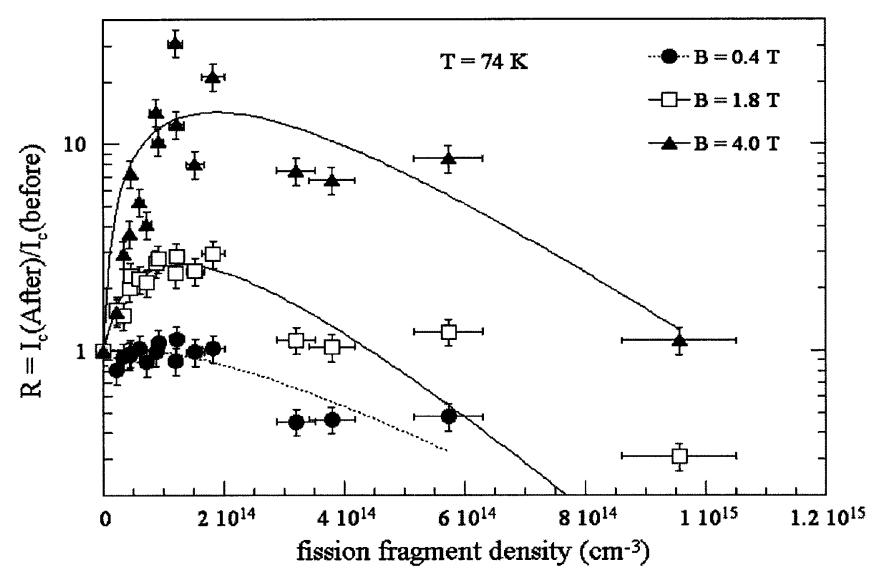

Fig. 1. $R$ vs. fission fragment density, $d . T=74 \mathrm{~K}$. Magnetic field is applied parallel to tape surface, and it varies; magnetic field values are shown in inset. It is seen that $R$ increases at low density, and then it decreases. $R$ increases with $B$. The standard deviation of $d$ is $\sim 10 \%$, whereas the standard deviation of $R$ is $\sim 20 \%$. Note that the $y$-axis is a log scale, which makes the peak appears less evident that it is in a linear scale plot.

magnetic fields. Magnetic field was applied parallel and perpendicular to the tape surface. Here, $I_{c}$ is defined by the $1 \mu \mathrm{V} / \mathrm{cm}$ criterion.

\section{EXPERIMENTAL DATA}

The average $I_{c}$ at $77 \mathrm{~K}$, zero applied field, before irradiation was $22 \mathrm{~A}$. This corresponded to a critical current density, $J_{c}$, of $\sim 2 \times 10^{4} \mathrm{~A} / \mathrm{cm}^{2}$. The standard deviation of $I_{c}$ was within $10 \%$ [9]. Let now define the ratio: $R=I_{c}$ (after)/ $I_{c}$ (before), where $I_{c}$ (after) and $I_{c}$ (before) are the critical currents after and before irradiation, respectively. $R$ is a function of fission fragment density, magnetic field, $B$, and temperature, $T$. Figs. 1 and 2 show a typical behavior of the $R$-vs.- $d$ curve. In Fig. $1, R$ is plotted versus the fission fragment density for varying applied magnetic fields at $T=74 \mathrm{~K}$. In Fig. 2, $R$ is plotted versus the fission fragment density for varying temperatures at $B=0.4 \mathrm{~T}$. Over the entire range of $B$ and $T$ investigated, all $R$-vs.- $d$ curves show the same behavior. The data collected may be phenomenologically described as follows. (1) At all temperatures and applied fields, there is a clear peak in $R$. (2) The fission fragment density at which $R$ peaks, $d_{\text {peak }}$, increases with $B$ and $T$. (3) The height of the peak, $R_{\text {peak }}$, depends on $B$ and $T$. In particular, $R_{\text {peak }}$ increases, in first approximation, exponentially with $B$ and $T$.

The fall-off shown in Figs. 1 and 2 is not only typical of the $\mathrm{U} / \mathrm{n}$ process, but also observed in other radiation experiments [7]. Although, most data we found in the literature are in the low range of defect density, $d<5 \times 10^{11} \mathrm{~cm}^{-3}$ [7], [8].

\section{Phenomenological Model}

It has been broadly noted in the literature that $R$ fall-off is due to reduction of the superconducting order parameter as indicated by a lowering of $T_{c}$ after irradiation [8]. Since the superconducting order parameter is a monotonic decreasing function of the ratio $\left(T-T_{c}\right) / T_{c}$ [6], its degradation due to the irradiation damage is larger at high $T$ then at low $T$. We note that if [8] is the case also the $R$ fall-off should be larger at high temperature than at low temperature. However, by comparing the rate

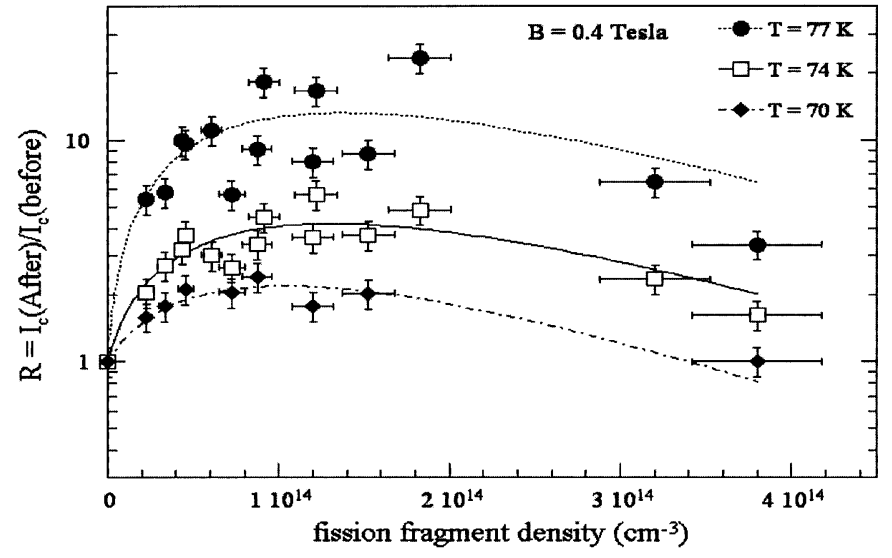

Fig. 2. $R$ vs. fission fragment density, $d$. A magnetic field of $0.4 \mathrm{~T}$ is applied perpendicular to the tape surface. Temperature varies; temperature values are shown in inset. $R$ increases with $T$. It is seen that $R$ increases at low density, and then it decreases. The standard deviation of $d$ is $\sim 10 \%$, whereas the standard deviation of $R$ is $\sim 20 \%$.

of $R$ fall-off, we observed that it is independent of the temperature. Furthermore, in the $\mathrm{U} / \mathrm{n}$ process, $T_{c}$ is reduced by only $\sim 2 \mathrm{~K} \mathrm{~K}$ at $d \sim 1.6 \times 10^{14} \mathrm{~cm}^{-3}$ [12], and this is insufficient to account for the observed fall-off. We take these observations as an indication that, in the range of investigation, the reduction in $T_{c}$ does not play a major role in the $R$ fall-off, and some other phenomena must be responsible for it.

To begin, we make the simple observation that $I_{c} \sim J_{c} \times$ $A$, where $A$ is the flow-area (active area of the HTS through which the supercurrent flows). Radiation defects are nonsuperconducting regions [1]-[4], Thus the presence of radiation-induced defects reduce the active flow-area. Because of the stochastic nature of the location of the damage, the percentage of undamaged flow-area is $\sim \exp \left(-V_{0} d\right)$, where $V_{0}$ is the volume of a single irradiation-induced defect.

In order to quantify the fall-off, we seek a phenomenological expression, which may capture, in a simple way, both the increase and the fall-off of $R$. We used:

$$
R=(1+f(B, T) d) \cdot \exp (-\alpha d)
$$

Where, $f(B, T)$ and $\alpha$ are two parameters to be determined experimentally by fitting the data.

\section{DISCUSSION}

The solid curves shown in Figs. 1 and 2, are obtained by fitting (2) to the data. The values of the parameter $\alpha$, and $f(B, T)$ for the best fitting curves were obtained by the process of least squares.

\section{A. The Parameter $\alpha$}

The parameter $\alpha$ was found to be independent on $B$ and $T$, across the entire range of $B$ and $T$. In other words, the value of $\alpha$, in all curves shown in Figs. 1 and 2, is about the same. The mean value of $\alpha$ was $4.1 \times 10^{-15} \mathrm{~cm}^{3}$, with standard deviation of $\sim 28 \%$. This finding supports the above hypothesis of $R$ fall-off being related to volume of the irradiation-induced defects. To check this hypothesis, we shall now compute $V_{0}$ and compare it to the fitting parameter $\alpha$. 
First, we note that the volume, $V_{0}$, of a single irradiation-induced defect, over which superconductivity is suppressed, is not merely the amorphous part of the defect. In fact, superconductivity is not fully restored until a distance of the order of the coherence length, $\xi$ [16], away from a nonsuperconducting region. Therefore, the volume over which superconductivity is destroyed is larger than just the geometric volume of the irradiation-induced damage. Herein, we consider $V_{0} \approx \pi\left(d_{d} / 2+\xi\right)^{2} l$, where $\xi$ is the coherence length (in Bi-2223, $\xi \approx 4 \mathrm{~nm}$ at $T=$ $77 \mathrm{~K}$ [17]), and $l$ is the quasicolumnar defect length $(\sim 2.7 \mu \mathrm{m})$, $d_{d} \sim 7.6 \mathrm{~nm}$ is the diameter of a fission fragment defect [10]. Using the above values, we obtained $V_{0} \sim 0.51 \times 10^{-15} \mathrm{~cm}^{3}$.

$V_{0}$, as calculated, differs to $\alpha$ by three standard deviations. However, we consider the discrepancy in magnitude between $\alpha$ and $V_{0}$ to be not such a bad disagreement, in particular, when we consider the uncertainty in the estimate of $V_{0}$, and the scatter in the data. A better estimate of damage size, when available, would be of course very useful to further test our model.

\section{B. The Parameter $f(B, T)$}

At this time we shall consider the parameter $f(B, T)$ merely as a fitting parameter, which phenomenologically take into account the enhancement of $I_{c}$ by irradiation. $f(B, T)$ varies with $T$, and with the magnitude and direction of $B . f(B, T)$ increases approximately exponentially with respect $T$ and $B$, and it varies in magnitude between $0.8 \times 10^{-14}$ to $200 \times 10^{-14} \mathrm{~cm}^{3}$, in the range of $T$ and $B$ here investigated. The fitting of (2) to the data shown in Fig. 1 gives $f(B, T) \sim 0.52 \times 10^{-14}$, $3.98 \times 10^{-14}, 19.8 \times 10^{-14} \mathrm{~cm}^{3}$ for $B=0.4,1.8$, and $4.0 \mathrm{~T}$, respectively. The fitting of (2) to the data shown in Fig. 2 gives instead $f(B, T) \sim 3.7 \times 10^{-14}, 7.2 \times 10^{-14}, 24.8 \times 10^{-14} \mathrm{~cm}^{3}$ for $T=70,74$, and $77 \mathrm{~K}$, respectively.

We observe that the fitting of the $R$-vs.- $d$ curves results in comparable values $f(B, T)$ when the experimental conditions (i.e., $B$ and $T$ ) are such that the ratios $B / B_{\text {irr }}$ have same values ( $B_{\text {irr }}$ is the irreversibility field prior irradiation at a given $T$; $B_{\text {irr }}$ values here used where derived by transport measurement as described in [9]). As an example, we point out that the value of $f(B, T)$, which gives the best fitting to the data at $74 \mathrm{~K}$ and $1.8 \mathrm{~T}$ in Fig. 1, is comparable to the value of $f(B, T)$, which gives the best fitting to the data at $70 \mathrm{~K}$ and $0.4 \mathrm{~T}$ in Fig. 2. Although $B$ and $T$ are different, the ratios $B / B_{\text {irr }}$ are in both cases $\sim 0.46$, i.e., $B_{\text {irr }} \sim 3.85 \mathrm{~T}$ at $74 \mathrm{~K}$ and $B$ parallel to the tape surface, and $\sim 0.87 \mathrm{~T}$ at $70 \mathrm{~K}$ and $B$ perpendicular to the tape surface.

This finding indicates that the experimental conditions, i.e., temperature, and magnetic field magnitude and direction, may all be represented by a single variable, i.e., $B / B_{\text {irr }}$.

\section{CONCLUSION}

We have shown that the reduction of the flow area may account for the $R$ fall-off; quantitatively the $R$ fall-off can be related to the size of the single irradiation-induced defect. If confirmed, this model suggests that a smaller size defect, although still in the shape of quasicolumnar defect, may result in a slower fall-off, hence may result in a higher $R_{\text {peak }}$. We note that this hypothesis is opposed to the generally accepted view that full-columnar defects provide the greatest $J_{c}$ enhancement, because they provide the largest pinning force [6]. However, this view is based only on vortex-damage interaction studies, and it neglects the reduction of flow-area effect. An effect which as shown here, may play a major role in determining the height of the $I_{c}$ enhancement peak.

In conclusion, these results suggest that a larger $J_{c}$ enhancement may be achievable, by more careful control of the single ion damage size. We also remind the reader that the results presented here are based on data on the $\mathrm{U} / \mathrm{n}$ process in $\mathrm{Ag} / \mathrm{Bi}-2223$ tape. It would be interesting try to extend the same analysis to other HTS, and other forms of radiation.

\section{ACKNOWLEDGMENT}

The authors wish to thank the staff of the Texas A\&M reactor where samples have been irradiated.

\section{REFERENCES}

[1] D. Bourgault, M. Hervieu, S. Bouffard, D. Groult, and B. Raveau, "3.5 GeV xenon ion irradiation effects in the superconducting oxide $\mathrm{YBa}_{2} \mathrm{Cu}_{3} \mathrm{O}_{7-X}(X<0.1)$ : a hrem investigation," Nucl. Instrum. Methods, vol. B 42, no. 61, 1989.

[2] M. Toulemonde, S. Bouffard, and F. Studer, "Swift Heavy Ions in Insulating and Conducting Oxides: Tracks and Physical Properties," Nucl. Instrum. Methods B, vol. 91, pp. 108-123, 1994.

[3] Y. Zhu, Z. X. Cal, R. C. Budhani, M. Suenaga, and D. O. Welch, "Structure and Effects of Radiation Damage in Cuprate Superconductors Irradiated with Several-Hundred-MeV Heavy Ions," Phys. Rev. B, vol. 6436, 1993.

[4] R. Hensel, B. Roas, S. Henke, R. Hopfengartner, M. Lippert, J. P. Strobel, M. Vildic, and G. Saemann-Ischenko, "Ion irradiation of epitaxial $\mathrm{YBa}_{2} \mathrm{Cu}_{3} \mathrm{O}_{7-X}$ films: Effects of electronic energy loss," Phys. Rev. B, vol. 48, p. 1125, 1990.

[5] D. Huang, Y. Sasaki, I. Hirabayashi, and Y. Ikuhara, "Long columnar defects with constant column size in 180-MeV Fe-irradiated $\mathrm{Bi}_{2} \mathrm{Sr}_{2} \mathrm{CaCu}_{2} \mathrm{O}_{7-X}$ crystals," Phys. Rev. B, vol. 61, p. $15442,2000$.

[6] H. Blatter, M. V. Feigel'man, V. B. Geshkenbein, A. I. Larkin, and V. M. Vinokur, "Vortices in high-temperature superconductors," Rev. Mod. Phys., vol. 66, p. 1125, 1994.

[7] L. Civale, "Irradiation processing for flux pinning enhancement in High- $T_{c}$ superconductor," in Processing and Properties of High- $T_{c}$ Superconductor: World Scientific, vol. 1, ch. 8.

[8] R. C. Budhani, "Studies of vortex localization columnar defects in high temperature superconductor," in Studies of High Temperature Superconductor, A. Narlikar, Ed: Nova Science Publiscers, 2000, vol. 33.

[9] A. Gandini, "Improvement of Critical Current, Irreversibility Field, and Anisotropy in BiSCCO Tape by the U/n Method," Ph.D., University of Houston, 2001.

[10] R. Weinstein, R.-P. Sawh, P. Drew, M. Murakami, T. Mocida, M. Chicamodo, G. Krabbes, and W. Biegar, Physica C, vol. 383, pp. 214-222, 2002.

[11] R. Weinstein, “An overview of U/n processing," in Proc. of 12th International Symposium of Superconductivity (ISS-99), Morioka, Japan, Oct. 17-19, 1999, pp. 521-526.

[12] A. Gandini, R. Weinstein, Y. R. Ren, R. P. Sawh, D. Parks, Y. C. Guo, B. Zeimetz, S. X. Dou, S. Tönies, C. Klein, and H. W. Weber, "Critical current enhancement in $(\mathrm{Bi}, \mathrm{Pb})_{2} \mathrm{Sr}_{2} \mathrm{Ca}_{2} \mathrm{Cu}_{3} \mathrm{O}_{10}$ tapes via isotropic quasicolumnar defects, induced by fission products," Physica $C$, pp. 1453-1454, 2000.

[13] S. X. Dou and H. K. Liu, "Ag-Sheathed Bi(Pb)SrCACuO superconducting tapes," Supercond. Sci. Technol., vol. 6, pp. 297-314, 1993.

[14] R. Weinstein, Interaction of Radiation and Matter, NY: McGraw Hill, 1966.

[15] S. Tönies, H. W. Weber, Y. C. Guo, S. X. Dou, R.-P. Sawh, and R. Weinstein, "Improved in-field behavior of uranium doped BiSCCO tapes by enhanced flux pinning," IEEE Transactions on Applied Superconductivity, vol. 11, pp. 3904-3907, Sept 2001.

[16] M. Tinkham, Introduction to Superconductivity: McGraw-Hill, Inc., 1996.

[17] P. Poole, Handbook of Superconductivity: Academic Press, 2000. 